\title{
Validación de constructo de dos instrumentos para evaluar el proceso de mediación en el aula para favorecer la inclusión educativa
}

\section{Validation of construct of two instruments to evaluate the mediation process in the classroom to ensure educational inclusión}

\author{
(iD) Rubén López Vázquez \\ CIFE- Secretaria de Educación Pública- Dirección de Educación Especial (México) \\ (iD) Sergio Tobón Tobón \\ Centro Universitario de Altos Estudios en Socioformación y Sociedad del Conocimiento, \\ Cuernavaca (México) \\ iD María Guadalupe Veytia Bucheli \\ Facultad de Ciencias de la Educación del Instituto de Ciencias Sociales y Humanidades \\ de la Universidad Autónoma del Estado de Hidalgo (México) \\ D Luis Gibran Juárez Hernández \\ Centro Universitario de Altos Estudios en Socioformación y Sociedad del Conocimiento, \\ Cuernavaca (México)
}

\section{Resumen}

En el contexto actual es necesario diseñar, elaborar, aplicar y verificar la validez de constructo de instrumentos para evaluar los procesos de mediación en el aula para favorecer la inclusión educativa acorde con la sociedad del conocimiento. El estudio tiene los siguientes objetivos: 1) aplicar una rúbrica para docentes y otra para estudiantes para analizar la validez del constructo teórico y 2) realizar la validez de constructo mediante el análisis factorial exploratorio (AFE). Se aplicó a 615 docentes y 645 estudiantes de escuelas públicas. Se realizó el AFE, utilizando el método de extracción de factorización de ejes principales. Los resultados identificaron un factor e incluyó las diez preguntas propuestas. En docentes explicó una varianza total de $75.225 \%$ y en estudiantes el $74.432 \%$. Se demostró la calidad de ambas rúbricas al obtener factores apropiados con cargas superiores a .5, lo que confirmó la validez del constructo teórico que mide. Asimismo, el instrumento de docentes presentó una alta confiabilidad de .967 y en estudiantes .965, medida por el Alfa de Cronbach. Se ha demostrado con los resultados la calidad de las dos rúbricas para evaluar la mediación didáctica en el aula para favorecer la inclusión educativa de los estudiantes de educación primaria en la Ciudad de México al haber obtenido unos índices de fiabilidad adecuados y confirmando la validez del constructo que mide, por lo que son viables de ser empleados por sus características métricas apropiadas.

\section{Abstract}

In the current context, it is necessary to design, develop, apply and verify the validity of the construction of tools to evaluate mediation processes in the classroom in order to promote educational inclusion in line with the knowledge society. The study has the following objectives: 1) to apply one rubric for teachers and another for students to analyze the validity of the theoretical construct and 2) to perform construct validity by exploratory factorial analysis (AFE). It was applied to 615 teachers and 645 students from public schools. AFE was carried out, using the method of extraction of factorization of main axes. The results identified one factor and included the ten proposals questions. Teachers explained a total variance of $75,225 \%$ and students $74,432 \%$. The quality of both headings was demonstrated by obtaining appropriate factors with loads higher than . 5, which confirmed the validity of the theoretical construct that measures. Likewise, the instrument of teachers presented a high reliability . 967 and in students . 965, measured by the Alfa of Cronbach. The results have demonstrated the quality of the two headings to evaluate didactic mediation in the classroom in order to promote the educational inclusion of primary school students in Mexico City, having obtained a adequate reliability and confirming the validity of the measuring construct, so they are viable to be used because of their appropriate metric characteristics.

\section{Palabras clave / Keywords}

inclusión educativa; proceso de enseñanza; rúbrica socioformativa; validación de constructo. educational inclusion; teaching process; socioformative rubric; construct validation. 


\section{Introducción}

Los cambios que surgen en la actualidad, trastocan en diversos ámbitos de la sociedad, sobre todo en las pautas de interacción de las nuevas generaciones, que se convierten en retos que requieren nuevas formas de actualización, enseñanza, aprendizaje y evaluación (Martín-Pastor \& Durán, 2019). Ante este desafío, es necesario transformar la práctica docente, de una enseñanza orientada a la transmisión y mecanización del conocimiento, a una con más énfasis en la formación integral, basado en la indagación contextual y en la creación de nuevos conocimientos. En este sentido, se promueve una acción bidireccional entre el mediador y el estudiante, basada en la coevaluación y autoevaluación para detectar oportunidades de mejora continua para el logro de las metas educativas (Herrera \& Tobón, 2017; Tourón, Martín, Navarro, Pradas \& Íñigo, 2018), en un marco de derechos que reconozca las características, pensar, hablar, actuar y soñar de los estudiantes (Cooc, 2019; Moriña \& Carballo, 2018; Polo \& Aparicio, 2018; Roose, Goossens, Vanderlinde, Vantieghem \& Avermaet, 2018; Tabatabaee, Motallebzadeh, Ashraf \& Baghaei, 2018).

El reto en estos tiempos no se reduce solamente a la centralización de la educación, es necesario dar mayor autonomía a la gestión del currículo, como reiteradamente se planeta (Ramos \& Castellanos, 2016). Una visión diferente de enseñar, aprender y evaluar (Gutiérrez, Herrera, Bernabé \& Hernández, 2016), en donde el eje rector sea el proceso de aprendizaje y formación integral del estudiante (Aspeé, González \& CavieresFernández, 2019; Vidal \& Manríquez, 2016). Mediante estrategias y acciones que favorezcan el desarrollo de competencias, para identificar, explicar, argumentar, abordar y resolver problemas de su entorno con responsabilidad ética, en un marco de trabajo colaborativo, respeto y valoración de la diversidad (Lara, Rebolledo \& Rojano, 2019; Tourón et al., 2018).

En este contexto, es importante abordar el constructo de la mediación en el aula, para fortalecer la inclusión educativa de la diversidad de estudiantes. Una mediación didáctica comprendida desde el enfoque de la socioformación, que implica que los estudiantes demuestren con evidencias concretas el desarrollo de competencias, mediante actuaciones colaborativas para identificar, interpretar y argumentar al abordar y resolver problemas del entorno (Castro-Rubilar, Castañeda-Díaz, Ossa-Cornejo, Blanco-Hadi \& CastilloValenzuela, 2017; Herrera \& Tobón, 2017). Procesos en donde la función del mediador, es organizar y generar ambientes pedagógicos pertinentes para facilitar una educación integral (López, Fuentes \& Pozo, 2019), con un plan ético de vida, dando lo mejor, para afrontar los desafíos actuales y contribuir al desarrollo de un mundo mejor (Naranjo, 2017; Ortega, Hernández \& Tobón, 2015; Santana-Vega, Medina-Sánchez \& FelicianoGarcía, 2019).

Para efectos de esta investigación, se aborda el concepto de mediación didáctica como las acciones de acompañar, promover y sistematizar la formación integral de los estudiantes para ser emprendedores, mediante estrategias, actividades y recursos pertinentes, dinámicas, flexibles y retadoras, mediante el trabajo colaborativo, valores universales, gestión del conocimiento y la metacognición. En este proceso, el mediador debe ser un puente articulador entre el contexto, los conocimientos del estudiante y el currículo, para hacer posible un aprendizaje significativo acorde con los retos actuales. (Gargallo, Morera, Iborra, Climent, Navalón, \& García, 2014; Parra, Tobón \& López, 2015; Tobón, Pimienta, Juárez \& Hernández, 2018; Tourón et al., 2018).

Rodorigo, Fernández-Larragueta \& Fernández-Sierra (2019) señalan que para alcanzar el éxito en la enseñanza para la diversidad, es necesario, que el docente se involucre con reglas y técnicas científicas que le brinden posibilidades de diseñar y desarrollar una planeación didáctica, con tareas de impacto acorde con las condiciones, características y procesos de aprendizaje de los estudiantes (Aspeé et al., 2019; Cherng \& Davis, 2017; Martín-Pastor \& Durán, 2019; Rikkert, Wim \& K. van Veen, 2018), minimizando así, la posibilidad de caer en la improvisación y en la mecanización de los conocimientos (Tourón et al., 2018). Promoviendo así, una mediación apropiada para la diversidad, acorde con la sociedad del conocimiento y con el contexto de los estudiantes (Tobón, 2017), para que busquen y descubran por sí mismos lo que desean y requieren realizar para el logro de las metas educativas (Muhonen, Pakarinen, Poikkeus, Lerkkanen \& Rasku-Puttonen, 2018; Villa, 2019).

Es relevante aprovechar estos procesos de cambios, como un producto de investigación, indicador tangible de intervención en el contexto escolar y áulico en la sociedad actual. Para identificar y minimizar las barreras que originan toda forma de exclusión en los procesos de participación y aprendizaje. Impulsando así, el derecho de los estudiantes a recibir una educación de calidad, pertinente a su contexto, características, necesidades y coherente con las metas educativas de la sociedad actual (Castro-Rubilar et al., 2017; Herrera \& Tobón, 2017). Este reto, implica una gran relevancia al constructo de la mediación didáctica, ya que puede limitar o impulsar la inclusión de los estudiantes, por lo que es necesario contar con instrumentos eficaces 
que la evalúen y den cuenta de los procesos de inclusión (Tourón et al., 2018). Al respecto, García (2015), refiere la necesidad de los centros educativos de contar con instrumentos que evalúen y den cuenta del proceso de inclusión, garantizando así la participación y aprendizaje en igualdad de oportunidades.

Numerosas investigaciones abordan la validación del constructo de la mediación didáctica articulada con otros constructos como las competencias (Luna \& Reyes, 2015); competencia digital (Tourón et al., 2018), creencias epistemológicas (Vizcaino, Manzano \& Casas, 2015); la práctica docente (Díaz, Fernández, Faouzi \& Henríquez, 2015); evaluación de la calidad académica (Domínguez \& Sanabria 2018), competencias profesionales (Mendoza \& Covarrubias, 2016), pero son pocas las que abordan y dan cuenta sobre instrumentos que evalúan los procesos de la mediación didáctica con enfoque inclusivo o socioformativo (Castro-Rubilar et al., 2017; Hernández, Tobón \& Guerrero, 2016), y más aún, que se apliquen al mismo tiempo con los estudiantes, es ahí en donde radica la importancia de la validación de constructo de los instrumentos, que valoren la mediación didáctica para asegurar la inclusión educativa de la diversidad de estudiantes (Liu, Ludu \& Holton, 2015; Lizasoain, Tourón \& Sobrino, 2015).

La originalidad de la investigación, radica en la formalización científica del análisis de los instrumentos con enfoque y estructura socioformativa (Tobón, 2017), que permitan obtener referencias desde la perspectiva docente y estudiante, sobre el proceso de mediación en el aula para favorecer la inclusión educativa. El presente estudio se enfocó en los siguientes objetivos: 1) aplicar los instrumentos a la población de estudio para analizar la validez del constructo teórico planteado y 2) realizar la validez de constructo mediante el análisis factorial exploratorio (AFE), utilizando el método de extracción de factorización de ejes principales.

\section{Metodología}

\subsection{Instrumentos}

Se diseñaron dos rúbricas, una para docentes y otra para estudiantes, con enfoque y estructura socioformativa, con 10 preguntas cada una (ver Tabla 1 y Tabla 2), los descriptores de calidad en las rúbricas, consideran cinco niveles de desempeño establecidos por el enfoque socioformativo (ver Tabla 3), permiten identificar la forma de responder las preguntas por los participantes a partir de la gestión del conocimiento (Gómez \& Tobón, 2018; López, Tobón, Veytia \& Juárez, 2019).

\section{Tabla 1}

Rúbrica para docentes

\section{Preguntas}

1. Inicio las clases con una planeación didáctica considerando las características de los estudiantes.

2. Inicio las clases con una introducción de lo que aprenderán y realizarán los estudiantes mediante estrategias motivantes.

3. Aplico estrategias didácticas para el desarrollo de competencias de todos los estudiantes.

4. Utilizo diversos materiales didácticos para el desarrollo de aprendizajes significativos de todos los estudiantes.

5. Trabajo de forma colaborativa para el logro de las metas educativas y favorecer la inclusión de todos los estudiantes.

6. Desarrollo las clases a partir del abordaje y solución de un problema del contexto mediante el trabajo colaborativo.

7. Diseño e implemento proyectos innovadores para desarrollar las clases y favorecer la inclusión de todos los estudiantes.

8. Promuevo en el aula acciones para el reconocimiento y valoración de las diferencias de los estudiantes, como una oportunidad de aprendizaje, para favorecer la inclusión.

9. Promuevo acciones en el aula para impulsar la formación en valores y el crecimiento personal.

10. Utilizo la evaluación para el mejoramiento continuo de los estudiantes, como una oportunidad de aprendizaje. 
Tabla 2

Rúbrica para estudiantes

\section{Preguntas}

1. Tu profesor inicia las clases de acuerdo con una planeación de actividades considerando tus características personales y de tus compañeros.

2. Al iniciar las clases te sientes motivado y sabes qué realizarás durante el día.

3. Tu profesor emplea actividades con juegos durante las clases para que te motives y todos aprendan.

4. Tu profesor utiliza diversos materiales que facilitan tu aprendizaje y la de tus compañeros.

5. Tu profesor trabaja en las clases de manera colaborativa con todos los estudiantes para que aprendan con mayor facilidad

6. Tu profesor trabaja en las clases a partir de la solución de un problema de tu comunidad.

7. Tu profesor trabaja en las clases a partir de proyectos que logren resolver problemas de tu comunidad.

8. Tu profesor en las clases motiva la participación de todos los estudiantes para que trabajen respetando las diferencias de todos.

9. Tu profesor promueve la formación en valores y el crecimiento personal

10. Tu profesor utiliza la evaluación para el mejoramiento continuo, como una oportunidad de aprendizaje.

\section{Tabla 3}

Niveles de desempeño de las rúbricas

\begin{tabular}{|c|c|c|c|c|}
\hline \multicolumn{5}{|c|}{ Descriptores de calidad y desempeño } \\
\hline $\begin{array}{c}\text { Preformal } \\
\text { (Muy bajo=1) }\end{array}$ & $\begin{array}{l}\text { Receptivo } \\
\text { (Bajo=2) }\end{array}$ & $\begin{array}{l}\text { Resolutivo } \\
\text { (Medio=3) }\end{array}$ & $\begin{array}{c}\text { Autónomo } \\
\text { (Medio alto=4) }\end{array}$ & $\begin{array}{c}\text { Estratégico } \\
\text { (Muy alto=5) }\end{array}$ \\
\hline $\begin{array}{l}\text { Desempeño sin } \\
\text { manejo de } \\
\text { nociones o } \\
\text { procedimientos. }\end{array}$ & $\begin{array}{l}\text { Desempeño } \\
\text { mecánico con } \\
\text { nociones } \\
\text { elementales. }\end{array}$ & $\begin{array}{l}\text { Desempeño } \\
\text { básico. Se aplica } \\
\text { lo esencial. }\end{array}$ & $\begin{array}{l}\text { Desempeño con } \\
\text { análisis, criterio y } \\
\text { argumentación. }\end{array}$ & $\begin{array}{c}\text { Desempeño con } \\
\text { liderazgo, vinculación, } \\
\text { interdisciplinariedad y } \\
\text { vinculación de saberes. }\end{array}$ \\
\hline
\end{tabular}

El proceso de validez de contenido de las rúbricas se realizó por 16 jueces con maestría o doctorado, expertos en la realización de valoraciones con ética e imparcialidad a la hora de emitir un resultado (Ford, 2018). Cada juez comunicó logros cuantitativos y cualitativos en cuanto a pertinencia del contenido y redacción de las rúbricas en cuatro grados, en donde 1 es el valor más bajo y 4 el valor más alto, presentados en un sólo formulario, con la finalidad de que tuvieran presente un comparativo de indicadores y descriptores. La validez de contenido se evaluó mediante la "V" de Aiken (Aiken, 1985), obteniendo los resultados (ver Tabla 4 y Tabla 5). El criterio para aceptar las dos rúbricas como válidos fue que presentaran una "V" Aiken mayor a .80.

\section{Tabla 4}

Validez de contenido del instrumento para docentes

\begin{tabular}{lcccccccccc}
\hline \multicolumn{10}{c}{ "V" Aiken } \\
\hline & 1 & 2 & 3 & 4 & 5 & 6 & 7 & 8 & 9 & 10 \\
Pertinencia & .958 & .958 & .937 & .958 & .958 & .979 & .958 & .916 & .937 & .958 \\
Redacción & .979 & .895 & .916 & .979 & .958 & .937 & .958 & .958 & .979 & .937 \\
\hline
\end{tabular}

Tabla 5

Validez de contenido del instrumento para estudiantes

\begin{tabular}{|c|c|c|c|c|c|c|c|c|c|c|}
\hline \multicolumn{11}{|c|}{ "V" Aiken } \\
\hline \multicolumn{11}{|c|}{ Preguntas } \\
\hline & 1 & 2 & 3 & 4 & 5 & 6 & 7 & 8 & 9 & 10 \\
\hline Pertinencia & .937 & .958 & 1 & .979 & .979 & .958 & .916 & .958 & .979 & .979 \\
\hline Redacción & .854 & .895 & .937 & .937 & .937 & .854 & .854 & .916 & .937 & .895 \\
\hline
\end{tabular}


Después se procedió con la aplicación a un grupo piloto de 50 docentes y 50 estudiantes de $4^{\circ}, 5^{\circ}$ y $6^{\circ}$ grado; se optó por estudiantes de estos grados, ya que la evaluación diagnóstica identificó que la mayoría de los estudiantes de $2^{\circ}$ y algunos de $3^{\circ}$ grado, aún se encontraban en proceso de consolidación de la lectura y escritura. Los datos obtenidos se analizaron mediante el coeficiente alfa de Cronbach a través del programa IBM-SPSS, versión 25, obteniendo los siguientes resultados: docentes .891 y en estudiantes .895. Tales resultados cumplen con los criterios mencionados por Nunnally \& Bernstein (1994), ya que establecen que los valores mínimos recomendados en los coeficientes de confiabilidad deben ser para investigaciones exploratorias por lo menos .70, para investigaciones básicas .80 y para toma de decisiones .90

Estos valores fluctúan entre 0 y 1 , en donde más cercano a 1 , mejor será la consistencia interna de los instrumentos (Salcines \& González, 2016). Se empleó el estadístico de consistencia interna Alpha de Cronbach (Cronbach, 1951, Salavera \& Usán, 2019), ya que es la más empleada por fijar una aplicación de las rúbricas y asume que si el mismo se dirige a medir un rasgo determinado, todas las preguntas que la integran deberían tener ese fin.

\subsection{Participantes}

Se gestionaron con cuatro directivos de escuelas primarias públicas de la Ciudad de México, para la aplicación de los instrumentos a docentes y 645 estudiantes de $4^{\circ}, 5^{\circ}$ y $6^{\circ}$ grado (ver Tabla 6 y Tabla 7). También se invitó a participar en la encuesta a docentes que asistían a los centros de actualización, por correo electrónico y redes sociales vía formulario Google creado por el CIFE, alcanzando una muestra de 615 docentes.

\section{Tabla 6}

Datos sociodemográficos de los docentes

\begin{tabular}{llcr}
\hline \multicolumn{1}{c}{ Características } & \multicolumn{3}{c}{ Datos } \\
\hline Sexo & Hombres & & Mujeres \\
Edad & $36.58 \%$ & & $63.42 \%$ \\
& $35-50$ & años & $30-50$ \\
Residencia & $96.70 \%$ & Urbana. & \\
& $1.10 \%$ & Semiurbana. \\
& $2.20 \%$ & Rural. & \\
Estudios & $7.52 \%$ & Normal básica & $23.45 \%$ \\
& $16.81 \%$ & Licenciatura & $32.30 \%$ \\
Experiencia docente & $4.64 \%$ & Maestría & $14.38 \%$ \\
Formación en inclusión & $.22 \%$ & Doctorado & $.66 \%$ \\
Condiciones económicas & $10-25$ & años & $10-30$ \\
& $42.69 \%$ & & $54.86 \%$ \\
\hline
\end{tabular}

Tabla 7

Datos sociodemográficos de los estudiantes

\begin{tabular}{llc}
\hline \multicolumn{1}{c}{ Características } & \multicolumn{2}{c}{ Datos } \\
\hline Sexo & Hombres & Mujeres \\
Promedio de edad & $53.33 \%$ & $46.66 \%$ \\
Residencia & & 9 a 11 Años. \\
\end{tabular}

\section{Validez de constructo mediante el análisis factorial exploratorio (AFE)}

La validez de constructo se analizó a través del AFE (Meroño, Calderón, Arias-Estero \& Méndez-Giménez, 2018a; Pineda, Castro \& Chaparro, 2018), con base en los resultados que se obtuvieron de la muestra. Se procedió a hacer una valoración del supuesto de correlación entre las variables, con el fin de validar si se justifica o no, su aplicación. Se empleó el índice Kaiser Meyer Olkin [KMO] (Calderón, Arias-Estero, Meroño 
\& Méndez-Giménez, 2018), el cual toma valores entre 0 y 1, con cargas superiores a .50 (las cargas menores a .50 se consideran inaceptables, de 5.5 a .59 pobres, .6 a .79 regulares y de .8 a 1 meritorios) y la prueba de esfericidad de Bartlett (Moliner et al., 2017). Este proceso verifica que la matriz de correlación sea la identidad. Se obtuvieron resultados adecuados por lo que se procedió a la aplicación del AFE, se utilizó esta técnica estadística multivariada ya que busca identificar una estructura subyacente de un mínimo de factores que expliquen la máxima cantidad de información contenida en las variables observadas -ítems- en base a la variabilidad común que hay entre ellas y los factores latentes -dimensiones- (García, Mayorga \& Barrera, 2019; Martínez \& González, 2018; Mavrou, 2015).

Finalmente, con los datos obtenidos de la población se llevó a cabo el análisis mediante el coeficiente Alfa de Cronbach (Cronbach, 1951), para obtener la consistencia interna de las rúbricas.

\section{Resultados}

En primer lugar, se presenta el AFE para el estudio de la dimensionalidad. Los resultados obtenidos de la aplicación de los instrumentos a 615 docentes y 645 estudiantes fueron analizados a través del programa IBM-SPSS, versión 25. Para confirmar su aplicación, se realizaron las pruebas de Kaiser-Meyer-Oikin y la prueba de esfericidad de Bartlett. La matriz de correlación muestra correlaciones estadísticamente significativas entre todas las preguntas evaluadas a docentes y estudiantes (ver Tabla 8 y Tabla 9). Los resultados obtenidos en el Índice KMO muestran un valor cercano a 1 en docentes y estudiantes, y en la prueba de esfericidad de Bartlett, muestra un nivel de significación menor de .5 (ver Tabla 10). Estos resultados fueron favorables, permitiendo que se realizara el AFE. De esta forma se eligió el método de extracción de factorización de ejes principales y el número de componentes a retener se basó en la norma de Gutman-Kaiser (Gorsuch, 1983). Se eligió el método de extracción de factorización de ejes principales, por ser un método iterativo y robusto, fundado en la extracción continua de aquellos componentes que revelan la mayor fracción de la varianza común para afrontar posibles violaciones del supuesto de normalidad, obteniendo correlaciones moderadas (De Winter \& Dodou, 2012; Mavrou, 2015).

Tabla 8

Matriz de correlaciones entre las preguntas para docentes

\begin{tabular}{ccccccccccc}
\hline Preguntas & 1 & 2 & 3 & 4 & 5 & 6 & 7 & 8 & 9 & 10 \\
\hline 1 & $\mathbf{1 . 0 0 0}$ & & & & & & & & & \\
2 & .765 & $\mathbf{1 . 0 0 0}$ & & & & & & & & \\
3 & .740 & .815 & $\mathbf{1 . 0 0 0}$ & & & & & & & \\
4 & .747 & .745 & .800 & $\mathbf{1 . 0 0 0}$ & & & & & & \\
5 & .677 & .754 & .750 & .719 & $\mathbf{1 . 0 0 0}$ & & & & & \\
6 & .732 & .767 & .760 & .739 & .745 & $\mathbf{1 . 0 0 0}$ & & & & \\
7 & .758 & .787 & .803 & .789 & .757 & .867 & $\mathbf{1 . 0 0 0}$ & & \\
8 & .712 & .744 & .772 & .730 & .717 & .740 & .767 & $\mathbf{1 . 0 0 0}$ & & \\
9 & .712 & .743 & .755 & .727 & .747 & .736 & .767 & .817 & $\mathbf{1 . 0 0 0}$ & \\
10 & .740 & .706 & .696 & .740 & .678 & .778 & .783 & .745 & .760 & $\mathbf{1 . 0 0 0}$ \\
\hline
\end{tabular}

Tabla 9

Matriz de correlaciones entre las preguntas para estudiantes

\begin{tabular}{ccccccccccc}
\hline Preguntas & 1 & 2 & 3 & 4 & 5 & 6 & 7 & 8 & 9 & 10 \\
\hline 1 & $\mathbf{1 . 0 0 0}$ & & & & & & & & & \\
2 & .684 & $\mathbf{1 . 0 0 0}$ & & & & & & & & \\
3 & .684 & .761 & $\mathbf{1 . 0 0 0}$ & & & & & & & \\
4 & .704 & .712 & .800 & $\mathbf{1 . 0 0 0}$ & & & & & & \\
5 & .640 & .735 & .748 & .722 & $\mathbf{1 . 0 0 0}$ & & & & & \\
6 & .696 & .775 & .781 & .759 & .769 & $\mathbf{1 . 0 0 0}$ & & & & \\
7 & .729 & .792 & .835 & .816 & .783 & .871 & $\mathbf{1 . 0 0 0}$ & & & \\
8 & .682 & .731 & .814 & .777 & .739 & .789 & .817 & $\mathbf{1 . 0 0 0}$ & & \\
9 & .704 & .736 & .787 & .790 & .767 & .769 & .817 & .862 & $\mathbf{1 . 0 0 0}$ & \\
10 & .665 & .619 & .621 & .690 & .628 & .686 & .707 & .686 & .692 & $\mathbf{1 . 0 0 0}$ \\
\hline
\end{tabular}


Tabla 10

Índice de KMO y prueba de esfericidad de Bartlett en docentes y estudiantes

$\mathrm{KMO}$ y prueba de Bartlett

Medida Kaiser-Meyer-Olkin de adecuación de muestreo

Docentes

.961

Estudiantes

Chi-cuadrado aproximado

Gl

6763.214

45

Sig.

.000

7063.314

45

.000

Las comunalidades mostraron valores apropiados, indicando su representación en el modelo factorial en docentes y estudiantes (ver Tabla 11). Los valores derivados de las pruebas de Kaiser-Meyer-Oikin, determinó el número de factores como autovalores mayores que 1. Esta regla reflejó una estructura factorial con un factor en ambas rúbricas de la varianza. En docentes explicó el $75.225 \%$ de la varianza total, presentó un valor propio inicial de $77.685 \%$ e incluyo las diez preguntas sugeridas, y el modelo factorial en estudiantes, explicó el $74.432 \%$ de la varianza total, mostró un valor propio inicial de $76.903 \%$ e incluyó las diez preguntas propuestas. Ambos factores con cargas superiores a .50, concordando con la propuesta teórica del constructo tratado. Estos valores se confirman con los gráficos de sedimentación (ver Figura 1 y Figura 2). Esto permitió observar los factores posibles que pueden estar y la influencia de cada uno de ellos respecto a los demás (Moliner et al., 2017). Estas respuestas factoriales se confirman con la matriz factorial obtenida en docentes y estudiantes (ver Tabla 12).

Tabla 11

Comunalidades en docentes y estudiantes

\begin{tabular}{|c|c|c|c|c|}
\hline \multirow{2}{*}{ Preguntas } & \multicolumn{2}{|c|}{ Docentes } & \multicolumn{2}{c|}{ Estudiantes } \\
\cline { 2 - 5 } & Inicial & Extracción & Inicial & Extracción \\
\hline 1 & .698 & .707 & .618 & .624 \\
\hline 2 & .760 & .766 & .702 & .710 \\
\hline 3 & .786 & .784 & .784 & .783 \\
\hline 4 & .736 & .744 & .752 & .765 \\
\hline 5 & .690 & .698 & .697 & .707 \\
\hline 6 & .794 & .777 & .799 & .798 \\
\hline 7 & .830 & .833 & .854 & .871 \\
\hline 8 & .748 & .746 & .808 & .799 \\
\hline 9 & .757 & .751 & .808 & .805 \\
\hline 10 & .727 & .717 & .594 & .582 \\
\hline
\end{tabular}

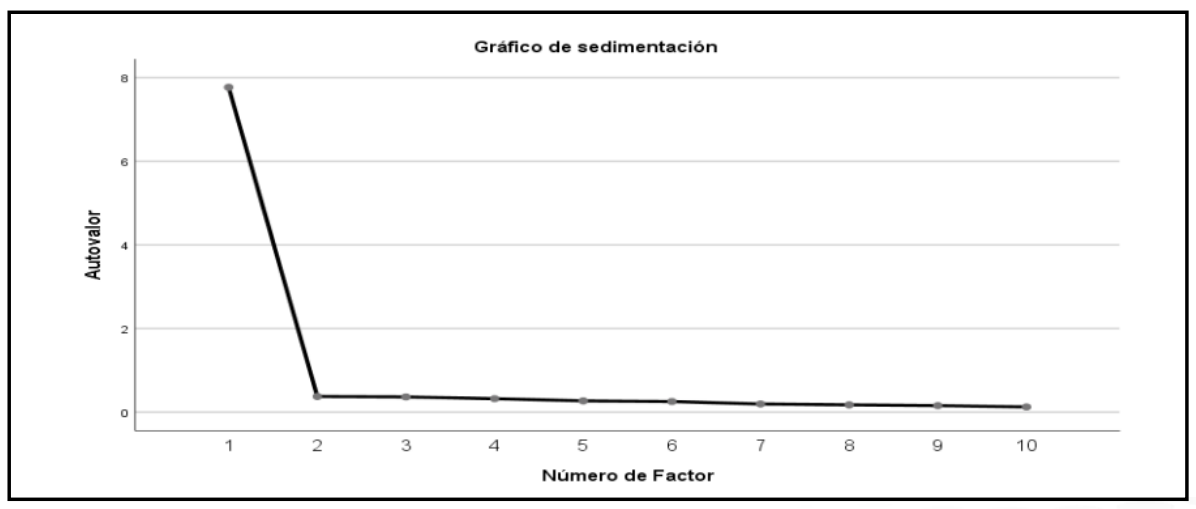

Figura 1. Gráfico de sedimentación en docentes. 


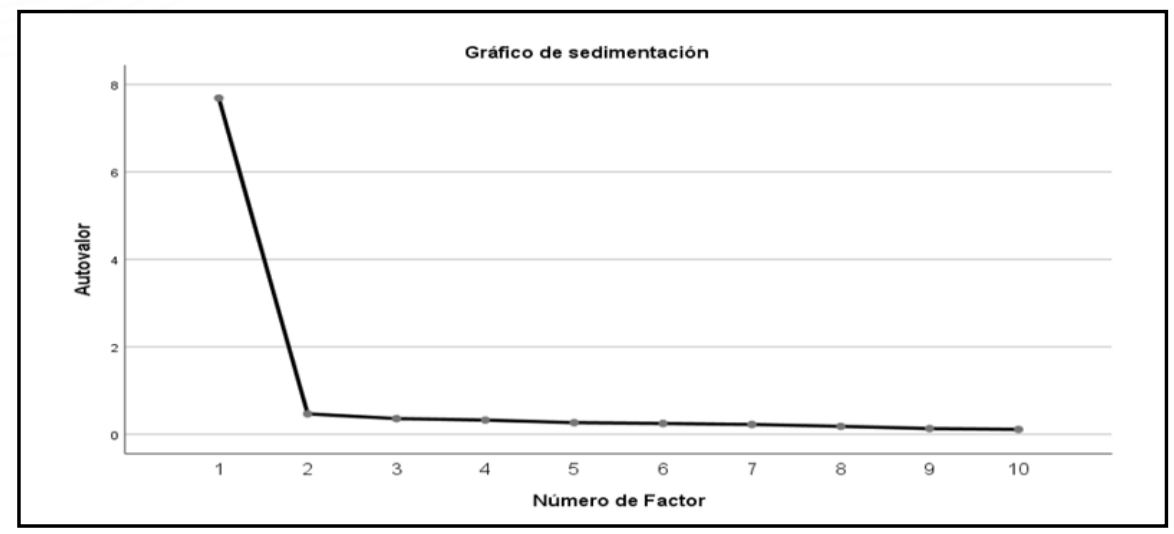

Figura 2. Gráfico de sedimentación en estudiantes

Tabla 12

Matriz factorial en docentes y estudiantes

\begin{tabular}{ccc}
\hline Preguntas & $\begin{array}{c}\text { Docentes } \\
\text { 1 Factor }\end{array}$ & $\begin{array}{c}\text { Estudiantes } \\
\text { 1 Factor }\end{array}$ \\
\hline 7 & .913 & .933 \\
3 & .885 & .897 \\
6 & .882 & .894 \\
2 & .875 & .893 \\
9 & .866 & .885 \\
8 & .864 & .875 \\
4 & .863 & .842 \\
10 & .847 & .841 \\
1 & .841 & .790 \\
5 & .835 & .763 \\
\hline
\end{tabular}

Por último, la rúbrica para docentes presentó una confiabilidad de .967 y en estudiantes presentó una confiabilidad de .965, medidas por el Alfa de Cronbach.

\section{Conclusiones}

Todo proceso de investigación es inacabado. Cada fase tiene su importancia y relevancia, que posibilitan continuar buscando alternativas para seguir colaborando con la sociedad. Ante este reto, se inició con un estudio de validez de contenido de dos instrumentos para evaluar el proceso de mediación en el aula para favorecer la inclusión educativa, antecedente a esta investigación de validez de constructo, en donde se concluyó que los instrumentos están redactados de manera adecuada y es comprensible por los potenciales usuarios, ya que los resultados obtenidos reflejaron una comprensión sobre las instrucciones, preguntas, descriptores de desempeño y los conceptos que se utilizaron en las rúbricas para docentes y estudiantes. La validez de contenido se evaluó mediante el coeficiente de la "V" de Aiken, obteniendo resultados mayores a .80, confirmando su validez. Por lo tanto, se les considera como instrumentos que evalúan la mediación didáctica y favorecen la inclusión de los estudiantes en el contexto áulico acorde con la sociedad del conocimiento. El Alfa de Cronbach para docentes, fue de .891 y en estudiantes .895, lo que confirmó su confiabilidad (Aguilar \& Sánchez, 2018).

Una segunda conclusión, las rúbricas validadas se fundamentan y estructuran mediante el enfoque y taxonomía socioformativa (Gómez \& Tobón, 2018; Tobón et al., 2018), estos brindan a docentes y estudiantes elementos más pertinentes y detallados, para evaluarse paso a paso con respecto a los procesos de mediación, participación, aprendizaje, desempeño, inclusión y compromiso con ellos mismos, con la finalidad de ir mejorando en forma continua (Bassi, Clerici \& Aquario, 2018; Carrasco, Alarcón, \& Trianes, 2018; Escudero, 2019; Utriainen, Tynjälä, Kallio \& Marttunen, 2018). Estos instrumentos representan una alternativa 
e innovación, ya que se centran en acciones esenciales para formar ciudadanos que desarrollen competencias para impulsar un desarrollo social sostenible, abordar y resolver problemas del contexto de forma idónea para el bien personal y de los demás, mediante el trabajo colaborativo, valores universales y un proyecto ético de vida, acorde con los retos actuales. El enfoque socioformativo ha justificado su eficacia en el contexto latinoamericano (Hernández et al., 2016; Tobón, 2018), obteniendo beneficios en el logro de las metas educativas. Propone cinco niveles de desempeño (ver Tabla 3) para medir y evaluar competencias en la sociedad, las organizaciones y demás sectores del ámbito educativo (Tobón, 2017a).

La confiabilidad de las rúbricas diseñadas acredita los elementos metodológicos esenciales que plantea el enfoque socioformativo como validadas, fiables y congruentes con el constructo (Vázquez, Tobón, Vázquez, Herrera \& Juárez, 2018), se pueden aplicar de forma simultánea o en diferentes momentos para evaluar el proceso de mediación en el aula para favorecer la inclusión educativa de la diversidad de estudiantes (Aspeé et al., 2019). Brindan la posibilidad de identificar los procesos de mediación didáctica que dan respuesta a las características, necesidades, intereses, ritmos y estilos de aprendizaje de los estudiantes, con la finalidad de transformar la gestión del currículum en un marco de respeto y tolerancia a la diversidad (Akram \& Zepeda, 2015; Meroño, Calderón, Arias-Estero \& Méndez-Giménez, 2018; Tobón, 2017).

Una tercera conclusión, las rúbricas que se validaron permiten a directivos y docentes evaluar, analizar y reflexionar sobre la mediación didáctica, que puede favorecer o dificultar el acceso, permanencia, aprendizaje, participación o promoción oportuna de los estudiantes en el proceso educativo. Favorecen la construcción de una escuela inclusiva, asegurando la igualdad de oportunidades y la plena participación de cada uno de los estudiantes (Kramarczuk, Fergus \& King, 2017). Impulsan una evaluación formativa basada en evidencias de desempeño y la colaboración entre todos los integrantes, como ejes esenciales para avanzar hacia sociedades más inclusivas y justas (Martínez, Tobón \& López, 2018).

Una cuarta conclusión, el AFE realizado a los datos obtenidos de la aplicación de las rúbricas, permitió identificar la existencia de un factor e incluyó las diez preguntas propuestos por los autores respectivamente. En docentes explico el $75.225 \%$ y en estudiantes el $74.442 \%$ de la varianza total en cada rúbrica. Se ha demostrado la calidad de ambas al obtener factores apropiados con cargas superiores a .5, lo que confirmo la validez del constructo teórico que mide.

Una quinta conclusión, ambas rúbricas presentan una alta confiabilidad, en docentes de .967 y estudiantes de .965 , medidas por el alfa de Cronbach. Ambos factores con cargas superiores a .50, concordando con la propuesta teórica del constructo tratado. Estos valores reflejan un grado adecuado de estabilidad y consistencia interna entre los ítems del instrumento.

Se ha demostrado con estos resultados la calidad de las rúbricas, que obtuvieron índices de fiabilidad adecuados, confirmando la validez del constructo que mide, por lo que son viables de ser empleados por sus características métricas apropiadas. Se puede plantear que los instrumentos son acordes con los avances teóricos, y pueden ser relevantes en futuras investigaciones (Vázquez et al., 2018). Por lo cual, deben ser confirmados por otras investigaciones que utilicen estos instrumentos en poblaciones similares a la educación primaria u otros niveles educativos públicos o privados, así como con otros instrumentos validados con relación a la mediación didáctica, con la finalidad de irlos ajustando de acuerdo con las necesidades y características de cada contexto.

\section{Referencias}

Aguilar, C. M., \& Sánchez, G. I. (2018). Construcción y validación de un instrumento para valorar desempeños pedagógicos de estudiantes en formación inicial. Revista Educación, 42(1), 2215-2644. doi:

10.15517/revedu.v42i1.22728

Aiken, L. R. (1985). Three Coeficients for Analyzing the Reliability and Validity of Ralings. Educatlonai and Psychologjcal Measurement, 45(0), 131-142. Available at: https://goo.gl/8P4WyW

Akram, M., \& Zepeda, S. (2015). Development and Validation of a Teacher Self-assessment Instrument. Journal of Research and Reflection in Education, 9(2), 134-148. Available at: https://goo.gl/UF6GmF

Aspeé, J., González, J., \& Cavieres-Fernández, E. (2019). Instrumento para medir el compromiso estudiantil integrando el desarrollo ciudadano, una propuesta desde Latinoamérica. Revista Complutense de Educación, 30(2), 399-421. doi: 10.5209/RCED.57518

Bassi, F., Clerici, R., \& Aquario, D. (2018). Evaluate the quality of didactics in the university: the opportunities offered by latent class modeling. The TQM Journal, 30(2), 168-180. doi: 10.1108/TQM-10-2017-0127

Calderón, A., Arias-Estero, J. L., Meroño, L., \& Méndez-Giménez, A. (2018). Diseño y Validación del Cuestionario de Percepción del Profesorado de Educación Primaria sobre la Inclusión de las Competencias Básicas (\#ICOMpri3). Revista complutense de educación: Estudios sobre Educación, 34(19), 67-97. doi: 10.15581/004.34.67-97 Carrasco, C., Alarcón, R., \& Trianes, M. V. (2018). Social Adjustment and Cooperative Work in Primary Education: Teachers' and Parents' View. Rev Psicodidáct, 23(1), 56-62. doi: 10.1016/j.psicod.2017.02.001 
Castro-Rubilar, F., Castañeda-Díaz, M. T., Ossa-Cornejo, C., Blanco-Hadi, E., \& Castillo-Valenzuela, N. (2017). Validación de la escala de autoadscripción inclusiva en docentes Secundarios Delaware Chile. Psicología educativa, 23(2), 105-113. doi: 10.1016/j.pse.2017.05.003

Cooc, N. (2019). Do Teachers Spend Less Time Teaching in Classrooms With Students With Special Needs? Trends From International Data. Educational Researcher, 48(5), 273-286. doi: 10.3102/0013189X19852306

Cronbach, L. J. (1951). Coefficient alpha and the internal structure of tests. Psychometrika, 16(3), 297-334. doi: 10.1007/BF02310555

Cherng, H.-Y. S., \& Davis, L. A. (2017). Multicultural Matters: An Investigation of Key Assumptions of Multicultural Education Reform in Teacher Education. Journal of Teacher Education, 70(3), 219-236. doi:

$10.1177 / 0022487117742884$

De Winter, J. C. F., \& Dodou, D. (2012). Factor recovery by principal axis factoring and maximum likelihood factor analysis as a function of factor pattern and sample size. Journal of Applied Statistics, 39(4), 695-710. doi:

10.1080/02664763.2011.610445

Díaz, E., Fernández, A., Faouzi, T., \& Henríquez, C. F. (2015). Validación del constructo subyacente en una escala de evaluación del impacto de la investigación educativa sobre la práctica docente mediante análisis factorial confirmatorio. Revista de Investigación Educativa, 33(1), 47-63. doi: 10.6018/rie.33.1.193521

Domínguez, L. C., \& Sanabria Á. E. (2018). Validez de constructo y confiabilidad del ROTA-Q para la evaluación de la calidad académica de las rotaciones clínicas en estudiantes de medicina. Educ Med, 20(2), 71-78. doi:

10.1016/j.edumed.2017.11.010

Escudero, T. (2019). Evaluación del profesorado como camino directo hacia la mejora de la calidad educativa. Revista de Investigación Educativa, 37(1), 15-37. doi: 10.6018/rie.37.1.342521

Ford, C. (2018). Effective practice instructional strategies: Design of an instrument to assess teachers' perception of implementation. Studies in Educational Evaluation, 56(0), 154-163. doi: 10.1016/j.stueduc.2017.12.004

García, G. (2015). Escalas prácticas inclusivas en educación básica: confiabilidad y validez en una muestra mexicana. Revista Latinoamericana de Inclusión Educativa, 9(1), 77-93. Recuperado de https://bit.ly/2JNWTrJ

García, D., Mayorga, J., \& Barrera, R. (2019). Cálculo de un indicador de calidad de vida básico para Bogotá por secciones censales mediante análisis factorial. Perspectiva Geográfica, 24(1). doi: 10.19053/01233769.7861 Gargallo, B., Morera, I., Iborra, S., Climent, M. J., Navalón, S., \& García, E. (2014). Metodología centrada en el aprendizaje. Su impacto en las estrategias de aprendizaje y en el rendimiento académico de los estudiantes universitarios. Revista española de pedagogía, 72(274), 415-435. Recuperado de https://bit.ly/36dxLnt Gómez, J. A., \& Tobón, S. (2018). Diseño y validación de un instrumento para evaluar el impacto de los proyectos formativos en educación básica en México. Revista Atlante: Cuadernos de educación y desarrollo, (en línea). Recuperado de https://bit.ly/2001JHR

Gorsuch, R. L. (1983). Factor Analysis (2 ${ }^{\mathrm{a}}$ ed.). Hillsdale, NJ: Lawrence Erlbaum Associates.

Gutiérrez, A., Herrera, L., Bernabé, M., \& Hernández, J. (2016). Problemas de contexto: un camino al cambio educativo. Ra Ximhai, 12(6), 227-239. Recuperado de https://bit.ly/30xqvAw

Hernández, J., Tobón, S., \& Guerrero, G. (2016). Hacia una evaluación integral del desempeño: las rúbricas socioformativas. Ra Ximhai, 12(6), 359-376. Recuperado de https://bit.ly/2wLAHsd

Herrera, S., \& Tobón, S. (2017). El Director Escolar desde el Enfoque Socioformativo. Estudio Documental mediante la Cartografía Conceptual. Revista de Pedagogía, 38(102), 164-194. Recuperado de http://goo.gl/GtL5E7

Kramarczuk, C., Fergus, E., \& King, K. A. (2017). Pursuing Equity: Disproportionality in Special Education and the Reframing of Technical Solutions to Address Systemic Inequities. Review of Research in Education, 41(1), 6187. doi: 10.3102/0091732X16686947

Liu, H., Ludu, M., \& Holton, D. (2015). Can K-12 Math Teachers Train Students to Make Valid Logical Reasoning? A Question Affecting 21st Century Skills. (331-353). In: Ge X., Ifenthaler D., Spector J. (eds) Emerging Technologies for STEAM Education. Educational Communications and Technology: Issues and Innovations. Springer, Cham, 411. doi: 10.1007 / 978-3-319-02573-5 18

Lara, E., Rebolledo, G., \& Rojano, J. (2019). Mejorando el aprovechamiento de las actividades colaborativas por pares de estudiantes utilizando tecnología educativa en matemática. Revista Complutense de Educación, 30(2), 44-460. doi: 10.5209/RCED.57597

Lizasoain, L., Tourón, J., \& Sobrino, A. (2015). La evaluación del profesorado español y el impacto del feedback en las prácticas docentes. Análisis de TALIS 2013. Revista Española de Pedagogía, 73(262), 465-482. Recuperado de https://bit.ly/32xOtxA

López, J., Fuentes, A., \& Pozo, S. (2019). Educación Inclusiva e Intercultural al Borde de la Frontera: La Escolarización del Colectivo Mena. Revista de Pedagogía, 39(105), 173-196. Recuperado de https://bit.ly/30Z0IXs

López, R., Tobón, S., Veytia, M. G., \& Juárez, L. G. (2019). Validación de dos instrumentos para evaluar el proceso de mediación en el aula para asegurar la inclusión educativa. Revista Dilemas contemporáneos: Educación, Política y Valores, O(3). Recuperado de https://bit.ly/2XuRGPr

Luna, E., \& Reyes, É. (2015). Validación de constructo de un cuestionario de evaluación de la competencia docente. Revista Electrónica de Investigación Educativa, 17(3), 13-27. Recuperado de https://bit.ly/2NZcpXf Martín-Pastor, E., \& Durán, R. (2019). La inclusión educativa en los programas bilingües de educación primaria: un análisis documental. Revista Complutense de Educación, 30(2), 589-604. doi: 10.5209/RCED.57871 
Martínez, J. E., Tobón, S., \& López, E. (2018). Acreditación de la calidad en instituciones de educación superior: retos pendientes en América Latina. Revista Dilemas Contemporáneos: Educación, Política y Valores, 2(9), 1-19. Recuperado de https://bit.ly/2wFIfOB

Martínez, P., \& González, C. (2018). Validez de contenido y consistencia interna de un cuestionario sobre el proceso de inserción socio-laboral desde la mirada del universitario. Revista Complutense de Educación: Estudios sobre Educación, 29(3), 35-52. doi: 10.5209/RCED.53721

Mavrou, I. (2015) Análisis factorial exploratorio: cuestiones conceptuales y metodológicas. Revista Lebrija de Lingüística Aplicada a la Enseñanza de las Lenguas, O(19), 71-80. Recuperado de https://goo.gl/XHZAQG

Mendoza, M., \& Covarrubias, C. (2016). Competencias profesionales movilizadas en el prácticum de los grados de magisterio: propuesta de un instrumento. Revista de Pedagogía, 37(100), 161-185. Recuperado de http://goo.gl/5m69h2 Meroño, L., Calderón, A., Arias-Estero, J. L., \& Méndez-Giménez, A. (2018). Primary school student and teacher perceptions of competency-based learning. Cultura y Educación, 30(1), 1-37. doi: 10.1080/11356405.2018.1436796 Meroño, L., Calderón, A., Arias-Estero, J. L., \& Méndez-Giménez, A. (2018a). Diseño y validación del cuestionario de percepción del profesorado de Educación Primaria sobre el aprendizaje del alumnado basado en competencias (\#ICOMpri2). Revista Complutense de Educación: Estudios sobre Educación, 29(1), 215-235. doi: 10.5209/RCED.52200 Moliner, L., Aguirre, A., Domenech, A., Vallet, T., Vallet, I., \& Alegre, F. (2017). Diseño, validación y análisis factorial exploratorio y confirmatorio de la escala de actitud Cohesiona para la evaluación de la eficacia de los talleres de habilidades cooperativas. Estudios pedagógicos, 43(1), 213-234. Recuperado de https://bit.ly/2Gg8X48 Moriña, A., \& Carballo, R. (2018). Profesorado universitario y educación inclusiva: respondiendo a sus necesidades de formación. Psicología Escolar e Educacional, SP. Número Especial, 87-95. doi: 10.1590/2175-3539/2018/053 Muhonen, H., Pakarinen, E., Poikkeus, A.-M., Lerkkanen, M.-K., \& Rasku-Puttonen, H. (2018). Quality of educational dialogue and association with students' academic performance. Learning and Instruction, 55(0), 67-79. doi: 10.1016/j.learninstruc.2017.09.007

Naranjo, G. B. (2017). El trabajo docente en el marco de las políticas de educación inclusiva. Aproximación a partir de un estudio de caso. CPU-e. Revista de Investigación Educativa, O(24), 99-124. Recuperado de http://goo.gl/CaZSdP Nunnally, J. C., \& Bernstein, I. H. (1994). Psychometric Theory (3a ${ }^{\mathrm{a}}$ Ed). Nueva York: Mc-Graw-Hill.

Ortega, M., Hernández, J., \& Tobón, S. (2015). Análisis documental de la gestión del conocimiento mediante la cartografía conceptual. Ra Ximhai, 11(4), 141-160. Recuperado de http://goo.gl/vSLGs3

Parra, H., Tobón, S., \& López, J. (2015). Docencia socioformativa y desempeño académico en la educación superior. Paradígma, 36(1), 42-55. Recuperado de http://goo.gl/D7SLz9

Pineda, C. A., Castro, J. A., \& Chaparro, R. A. (2018). Estudio psicométrico de las Escalas de Bienestar Psicológico de Ryff en adultos jóvenes colombianos. Pensamiento Psicológico, 16(1), 45-55. Recuperado de https://bit.ly/2Z0EU8c Polo, M. T., \& Aparicio, M. (2018). Primeros pasos hacia la inclusión: Actitudes hacia la discapacidad de docentes en educación infantil. Revista de Investigación Educativa, 36(2), 365-379. doi: 10.6018/rie.36.2.279281

Ramos, A. L., \& Castellanos, P. J. (2016). Descentralización educativa y desarrollo en el estado de Oaxaca. Revista Iberoamericana para la Investigación y el Desarrollo Educativo, 7(13), $293-324$. Recuperado de https://goo.gl/zMoxBH Rikkert, M. van der Lans, Wim, J. C. M. van de Grift, \& K. van Veen (2018) Developing an Instrument for Teacher Feedback: Using the Rasch Model to Explore Teachers' Development of Effective Teaching Strategies and Behaviors. The Journal of Experimental Education, 86(2), 247-264. doi: 10.1080/00220973.2016.1268086

Rodorigo, M., Fernández-Larragueta, S., \& Fernández-Sierra, J. (2019). La Mediación intercultural como herramienta de inclusión: análisis de una experiencia escolar. Revista Complutense de Educación, 30(3), 747-761. doi:

10.5209/rced.58885

Roose, I., Goossens, M., Vanderlinde, R., Vantieghem, W., \& Avermaet, P. (2018). Measuring professional vision of inclusive classrooms in secondary education through video-based comparative judgement: An expert study. Studies in Educational Evaluation, 56(0), 71-84. doi: 10.1016/j.stueduc.2017.11.007

Salavera, C., \& Usán, P. (2019). Propiedades psicométricas del cuestionario QEWB de bienestar eudaimónico en adolescentes. Psicología Educativa, 25(0), 139-146. doi: 10.5093/psed2019a3

Salcines, I., \& González, N. (2016). Diseño y Validación del Cuestionario Smartphone y Universidad. Visión del Profesorado (SUOL). Revista complutense de educación: Estudios sobre Educación, 27(2), 603-632. doi: 10.5209/rev_RCED.2016.v27.n2.46912

Santana-Vega, L., Medina-Sánchez, P., \& Feliciano-García, L. (2019). Proyecto de vida y toma de decisiones del alumnado de Formación Profesional. Revista Complutense de Educación, 30(2), 423-440. doi: 10.5209/RCED.57589 Tabatabaee, M., Motallebzadeh, K., Ashraf, H., \& Baghaei, P. (2018). Development and Validation of a Teacher Success Questionnaire Using the Rasch Model. International Journal of Instruction, 11(2), 129-144. doi: 10.12973/iji.2018.11210a Tobón, S. (2017). Essential axes of knowledge society and socioformation. Mount Dora (USA): Kresearch. doi: 10.24944/isbn.978-1-945721-19-9

Tobón, S. (2017a). Proyecto de Enseñanza y evaluación con rúbricas. Mount Dora (USA): Kresearch Corp.

Tobón, S. (2018). El Proyecto de Enseñanza: Aprendizaje y evaluación. México: Centro universitario CIFE. Recuperado de https://goo.gl/fjxwVv

Tobón, S., Pimienta, J. H., Juárez, L. G., \& Hernández, J. S. (2018). Design and Validity of a Rubric to Evaluate Pedagogical Practices with a Socioformative Approach. Information, in press. Recuperado de https://bit.ly/30ZRPs9 
Tourón, J., Martín, D., Navarro, E., Pradas, S., \& Íñigo, V. (2018). Validación de constructo de un instrumento para medir la competencia digital docente de los profesores (CDD). Revista Española de Pedagogía, 76(269), 25-54. doi: 10.22550/REP76-1-2018-02

Vázquez, J., M., Tobón, S., Vázquez, J., Herrera, S. R., \& Juárez, L. G. (2018). Diseño y validez de contenido de una rúbrica socioformativa para evaluar el informe de prácticas profesionales en la Educación Normal. Revista Espacios, Número especial. CITED 2017, 29- 40. Recuperado de https://bit.ly/2GfoYHP

Vidal, D., \& Manriquez, L. (2016). El docente como mediador de la comprensión lectora en universitarios. Revista de la Educación Superior, 45(1), 95-118. doi: 10.1016/j.resu.2016.01.009

Villa, A. (2019). Liderazgo: una clave para la innovación y el cambio educativo. Revista de Investigación Educativa, 37(2), 301-326. doi: 10.6018/rie.37.2.365461

Vizcaino, A. E., Manzano, M., \& Casas, G. (2015). Validez de constructo y confiabilidad del Cuestionario de Creencias Epistemológicas sobre la Matemática en alumnos de secundaria básica. Revista Colombiana de Psicología, 24(2), 301316. doi: $10.15446 /$ rcp.v24n2.43974

Utriainen, J., Tynjälä, P., Kallio, E., \& Marttunen, M. (2018). Validation of a modified version of the Experiences of Teaching and Learning Questionnaire. Studies in Educational Evaluation, 56(0), 133-143. doi:

10.1016/j.stueduc.2017.12.007 